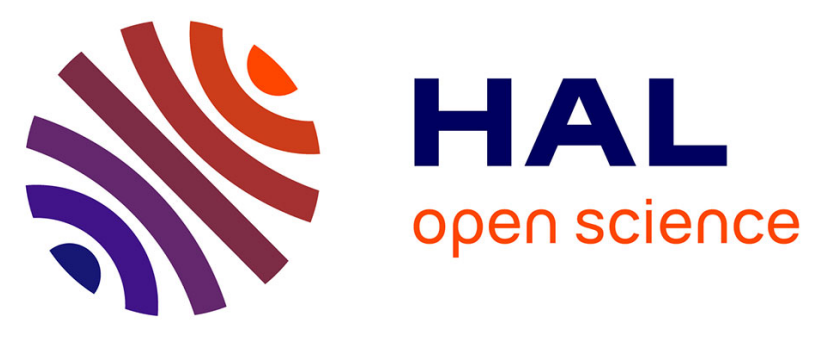

\title{
Fluid modelling of Stimulated Raman Scattering accounting for trapped particles benchmarked against fully kinetic simulations
}

Guillaume Tran, Pascal Loiseau, A Fusaro, Anne Héron, Stefan Hüller, L Maëder, P.-E Masson-Laborde, Denis Penninckx, G Riazuelo

\section{To cite this version:}

Guillaume Tran, Pascal Loiseau, A Fusaro, Anne Héron, Stefan Hüller, et al.. Fluid modelling of Stimulated Raman Scattering accounting for trapped particles benchmarked against fully kinetic simulations. Physics of Plasmas, 2020, 27 (12), pp.122707. 10.1063/5.0018669 . hal-03033346

\section{HAL Id: hal-03033346 https://hal.science/hal-03033346}

Submitted on 1 Dec 2020

HAL is a multi-disciplinary open access archive for the deposit and dissemination of scientific research documents, whether they are published or not. The documents may come from teaching and research institutions in France or abroad, or from public or private research centers.
L'archive ouverte pluridisciplinaire HAL, est destinée au dépôt et à la diffusion de documents scientifiques de niveau recherche, publiés ou non, émanant des établissements d'enseignement et de recherche français ou étrangers, des laboratoires publics ou privés. 


\title{
Fluid modelling of Stimulated Raman Scattering accounting for trapped particles benchmarked against fully kinetic simulations
}

\author{
G. Tran, ${ }^{1,}$ a) P. Loiseau, ${ }^{1}, 2$ A. Fusaro, ${ }^{1}$ A. Héron, ${ }^{3}$ S. Hüller, ${ }^{3}$ L. Maëder, ${ }^{1}$ P.-E. Masson-Laborde, ${ }^{1,2}$ \\ D. Penninckx, ${ }^{4}$ and G. Riazuelo ${ }^{1}$ \\ ${ }^{1)}$ CEA, DAM, DIF F-91297 Arpajon, France \\ 2) Université Paris-Saclay, CEA, LMCE, 91680 Bruyères-le-Châtel, France \\ ${ }^{3)}$ Centre de Physique Théorique (CPHT), CNRS, IP Paris, École Polytechnique, 91128 Palaiseau, \\ France \\ ${ }^{4)}$ CEA, CESTA, F-33116 Le Barp, France
}

(Dated: November 27, 2020)

A new fluid model describing backward stimulated Raman scattering (SRS) is presented based on parametric three-wave coupling in multidimensional geometry. It takes into account kinetic effects in the description of the plasma wave via a non linear frequency shift due to trapped electrons. The model is valid in the regime of hot and weakly inhomogeneous plasmas under conditions relevant for inertial confinement fusion with the plasma parameter $k_{L} \lambda_{D e} \geqslant 0.25$ ( $k_{L}$ standing for the plasma wave number and $\lambda_{D e}$ for the Debye length). Benchmarks of the model have been performed against the Maxwell-particle-in-cell (PIC) code EMI2D in order to calibrate the adjustable parameters controlling the non linear frequency shift. Two major configurations have been tested, one in a homogeneous plasma, with the onset of laser pump depletion and the other in an inhomogeneous plasma, producing auto-resonant growth. Good agreement between fluid and PIC simulations has been found for both configurations, in particular for the growth of SRS, and further on in time for the average backscatter level. The model is a promising tool to be implemented in multi-dimensional laser-plasma interaction packages coupled to hydrodynamics codes in order to compute SRS in mm-size volumes, usually inaccessible with PIC codes.

Keywords: Raman, Scattering, ICF, Reduced Model, Non Linear effects, Kinetic effects

\section{INTRODUCTION}

Experiments conducted on the US National Ignition Facility $(\mathrm{NIF})^{1}$ and on the Ligne d'Intégration Laser (LIL) facility in France ${ }^{2}$ exhibited very large levels of stimulated Raman backscattering (SRS) - up to $50 \%$ in the NIF's inner cones - being far above the estimations from predictions based on linear theory. SRS is a parametric instability ${ }^{3}$, in which a coherent electromagnetic (EM) 'pump' wave, characterized by its frequency and wave vector $\left(\omega_{0}, \mathbf{k}_{\mathbf{0}}\right)$, scatters off an electron plasma wave $\left(\omega_{L}, \mathbf{k}_{\mathbf{L}}\right)$ (or 'Langmuir' wave), resulting in a scattered EM light wave $\left(\omega_{R}, \mathbf{k}_{\mathbf{R}}\right)$. This three-wave coupling process satisfies the following matching conditions for frequencies and wave vectors:

$$
\omega_{0}=\omega_{R}+\omega_{L} \quad, \quad \mathbf{k}_{\mathbf{0}}=\mathbf{k}_{\mathbf{R}}+\mathbf{k}_{\mathbf{L}}
$$

As other laser-plasma instabilities, SRS is highly detrimental for inertial confinement fusion (ICF), for both the direct and indirect drive approaches, in the sense that it modifies the laser energy deposition needed to achieve an efficient fuel compression, enabling ignition. Although SRS in the context of ICF has been extensively studied over several decades, the modelling of SRS is still an unsolved issue to find reliable assessments of the amount of the scattered light, and of the level of the plasma wave

a)Electronic mail: guillaume.tran@cea.fr. amplitude that supports the instability, beyond a ps time scale and for mm-size plasma volumes needed for modeling realistic laser beams. Besides the difficulty of the various spatial and temporal scales involved, it is the non-linear evolution of laser-driven electron plasma wave that constitutes a major problem due to the complexity of the different processes that take place and their adequate modelling.

The different nonlinear regimes of electron plasma waves (EPW) can be classified using the $k_{L} \lambda_{D} \sim v_{\text {th,e }} / v_{\phi}$ plasma parameter, where $\lambda_{D}$ is the Debye length, $v_{\text {th,e }}$ the electron thermal velocity and $v_{\phi}$ the Langmuir wave phase velocity. Generally, for values $k_{L} \lambda_{D}>0.25$ the likelyhood of trapping of electrons in the potential wells of the wave increases. For this reason, this regime is often denoted 'kinetic regime' of electron plasma waves ${ }^{4,5}$, while the regimes with $k_{L} \lambda_{D}<0.15$ and with $0.15 \leq$ $k_{L} \lambda_{D} \leq 0.25$ are associated with processes, like the Langmuir decay instability and cascading ${ }^{6,7}$, related to strong and weak turbulence, respectively, although kinetic effects may also be present. These two regimes are generally described with models based on fluid-type equations. In the context of ICF, for electron temperatures $1-3 \mathrm{keV}$ and electron densities in the range $3 \%$ to $15 \%$ of the critical density $n_{c}$ for a $351 \mathrm{~nm}$ laser wavelength, the value of $k_{L} \lambda_{D}$ is situated in the interval $0.25 \ldots 0.45$, for which linear theory predicts a strong increase of Landau damping ${ }^{8}$ with $\left(k_{L} \lambda_{D}\right)^{2}$, eventually preventing the common EPW mode to develop (so called Landau cutoff ${ }^{9}$ ).

Electron trapping in the potential wells of the EPW leads to a deformation of the electron distribution func- 
tion around the wave phase velocity $v_{\phi}$, which results in a change of the EPW dispersion. The latter arises, due to the flattening of the distribution around $v_{\phi}$, in a decrease of the Landau damping, and in a non linear shift of the wave frequency. As elaborated in Refs. ${ }^{10-12}$, a frequency shift results, with a negative sign with respect to $\omega_{L}$ of the linear dispersion, that depends on the wave amplitude, which is therefore denoted as non linear frequency shift. Both the change in the Landau damping and the non linear frequency shift appear to be crucial for the understanding of SRS. Their study and modelling is challenging mainly because of the fast evolution towards non linear regimes related to the important modification of the electron distribution function.

Before describing our approach, and in order to highlight our work, we propose a brief review of the main results obtained in the field of the non linear saturation of SRS, starting by large amplitude waves and trapping. Frequency shift as a consequence of trapping was investigated first by the seminal papers from Morales and $\mathrm{O}^{\prime} \mathrm{Neil}^{12}$ and Dewar ${ }^{10,11}$, and revisited exhaustively with the help of mono-dimensional (1D) Vlasov simulations by Berger et al. ${ }^{13}$. They showed that this non linear frequency shift $\delta \omega^{\mathrm{NL}}$ is proportional to the square root of the wave electrostatic potential $\phi$, or equivalently to the wave amplitude $\delta n_{e}: \delta \omega^{\mathrm{NL}}=\left|\omega-\omega_{L}\right|=\eta \omega_{L} \sqrt{\delta n_{e} / N_{0}}$, where $N_{0}$ here is the local equilibrium density (see equation (9) further in the text). From analytic theory, the parameter $\eta$ results in the following form:

$$
\eta=\eta_{e} v_{\phi}^{3}\left(\frac{\partial^{2} f_{0}}{\partial v^{2}}\right)_{v_{\phi}},
$$

where $f_{0}(v)$ is the electron distribution function, disregarding fast oscillations of electrons in the wave electric field. The numerical factor $\eta_{e}$ depends on the way used to initialize the wave, either suddenly or adiabatically, whose value takes respectively 0.823 or 0.544 . Note that in Refs. ${ }^{10,12}$, an initial Maxwellian distribution is assumed. But this assumption it not valid on a longer time scale, because of the heating of the overall distribution function, as it can be easily checked in kinetic simulations. The parameter $\eta$ is therefore a parameter that, on a slow temporal scale, adjusts to the evolution of the average distribution function, and therefore is not constant in time. Its value obtained from the above expression for $f_{0}$ with the initial Maxwellian yields usually merely a lower bound. Also, it is worth noting that a non linear damping is associated to the frequency shift.

This fundamental work is particularly relevant to laser fusion research where we expect large amplitude EPWs excited by SRS. In this context, the laser-driven Langmuir wave evolution was investigated with the help of kinetic simulations using Maxwell-'particle-in-cell' (PIC) and reduced-PIC (RPIC) simulations ${ }^{4,14-20}$ or MaxwellVlasov codes ${ }^{21-24}$, in one and higher spatial dimensions. In particular, $\mathrm{Vu}$ et al. ${ }^{4}$ established the so-called kinetic inflation corresponding to a trapping induced decrease of Landau damping, leading to larger EPWs am- plitudes that saturate by dephasing due to non linear frequency shift. This work was extended by taking collisions into account ${ }^{25}$ and later in 1D PIC simulations ${ }^{26}$, modifying the inflation threshold. Then Yin and coworkers extended this work to two- (2D) and threedimension (3D), first in different $k_{L} \lambda_{D}$ regimes $^{14}$ exhibiting beam acoustics modes in the trapping regime, then showing the bowing ${ }^{15,20}$ of EPW resulting from non spatially-uniform trapping and trapped particle modulational instability, further addressing the threshold of trapping $^{16}$, the destabilization ${ }^{17,18}$ of under-threshold speckles by higher intensities speckles, and detrapping ${ }^{19}$ mechanisms by side-loss or collisions. Note that B-field generation due to Weibel instability has been identified in [20]. In addition, saturation of SRS by sideband instability was investigated in 1D and 2D with MaxwellVlasov simulations ${ }^{21-23}$. This instability was also investigated with 1D PIC code ${ }^{27}$. Non linear kinetic effects were investigated also in the context of backward Raman amplification $^{28-30}$ with 1D Vlasov code, leading to analytic expressions for the nonlinear damping and non linear frequency shift. Well-suited for dedicated studies in homogeneous plasmas, typically the length of one speckle and few ps, the investigation of SRS with kinetic codes is out of reach for describing realistic mm-large focal spot in mm-long plasmas on long time scale (typically hundreds ps), particularly when a multi-dimensional approach is required.

The stability of a large amplitude EPW in a homogeneous plasma is in itself a rich topic, but considering a more realistic plasma with spatial inhomogeneity adds even new effects. It is known that the action of trapped particles can destabilize the EPW, and therefore enhance SRS by allowing for growth in an inhomogeneous plasma slab beyond the resonance region bounded by the turning points. This broadening of the resonance region is due to spatial autoresonance ${ }^{31-33}$ and has to be accounted for in a realistic model. Note that autoresonance due to trapped electrons compensates the dephasing of the plasma inhomogeneity for profiles increasing along the propagation direction, which is different from nonlinear detuning mechanisms originating from harmonic generation $^{27,34,35}$ and relativistic effects ${ }^{36}$.

Many of the studies mentioned are 1D, and although the non linear detuning and damping depend on the local parameters, 2D effects have also proven to be of first importance: wave front bowing, multispeckles laser beam, seeding of neighboring speckles, side losses due to escaping trapped particles, transverse modulations and possibly EPW filamentation ${ }^{37}$. Because kinetic codes are not suitable for routinely simulate multidimensional ICF relevant plasmas on a long time scale (tens of ps), we aim at developing another way that we detail in this article. Our goal is to model the nonlinear development of SRS in an inhomogeneous plasmas by means of a nonlinear wave coupling which describes the resonant energy exchange between the incident laser beam, the reflected light beam and the EPW. The validity of 
our model is assessed by comparison to PIC simulations on well-controlled test problems. Such a three-wave coupling model for inhomogeneous plasmas in $2 \mathrm{D} / 3 \mathrm{D}$ is compatible with mesoscopic laser interaction codes such as PF3D $^{38}$, HARMONY ${ }^{39}$ and HERA ${ }^{40,41}$. Although this article will focus on 2D physics, we recall that our model was first elaborated in $1 \mathrm{D}^{42}$, allowing to elaborate the numerical scheme, and to check that classical results, such as the Rosenbluth amplification, auto-resonance and nonlinear frequency shift, are recovered. Such reduced modeling has already been attempted in numerous publications, usually in $1 \mathrm{D}^{4,20,25,28,30,43}$ with some variations regarding the non linear frequency shift parameter. We also emphasize the long-standing work from Bénisti and coworkers summarized in [44] where they develop a multidimensional three-wave coupling model. In contrast to this work, our model equations have been derived in applying consequently the linear dispersion relations, which is adequate in the context of driven plasma waves. For this reason, all potentially arising non linear wave features will occur in the wave amplitudes and not in the local wave coefficients as the wave numbers. Comparing all those models together is largely beyond the scope of this paper.

The present work is mainly focused on the nonlinear frequency shift arising from trapped particles. But detrapping mechanisms may occur in order to restore the Mawxellian electron distribution function, such as collisions and side-losses ${ }^{13,45}$. Collisions are naturally included in the EPW damping term of the Langmuir wave propagator and may increase the instability threshold or help restoring a Mawxellian EDF. However, it has been shown that for low-Z ICF plasmas, side-loss from speckles is the dominant mechanism ${ }^{45}$. Collisions are neglected throughout this paper both in fluid and PIC simulations. Side-loss becomes significant when the time to escape the width of a laser speckle, given approximately by $\tau_{\text {side }} \sim\left(f_{\#} \lambda_{0}\right) / v_{\text {th }}$, is shorter or similar to the bounce period, $\tau_{B}=2 \pi / \omega_{B}$ of trapped electron, with $v_{\text {th }}$ denoting the electron thermal speed and $\omega_{B} \sim \omega_{p e}\left|\delta n_{e} / N_{0}\right|^{1 / 2}$ the bounce frequency. The resulting criterion for the onset of side loss can be expressed as a condition for the Langmuir density perturbations, $\left|\delta n_{e} / N_{0}\right| \leq\left(k_{0} \lambda_{D} / f_{\#}\right)^{2}$ which is consistent with the criterions given in [45]. Inspecting this criterion one finds that side loss would appear only for density perturbation much smaller than the ones observed in our simulations, while it could be a more pertinent effect for higher temperatures and lower densities (say for $T_{e}>4 \mathrm{keV}$ and $N_{0} / n_{c} \leq 0.05$ ). Therefore, we neglect both detrapping effects. Note that side-loss and collisions may also be taken into account in our model ${ }^{46}$.

We turn now to the key point of our work, that is estimating the electron distribution function modifications for evaluating the non linear EPW frequency shift and Landau damping, without consistently resolving $f_{0}$ with kinetic simulations. On this basis, for a given value of $\eta$, we develop a model that includes the non linear frequency shift $\delta \omega^{\mathrm{NL}}$ of EPWs in the 3-wave coupling for SRS, by calculating $\left(\partial^{2} f_{0} / \partial_{v}^{2}\right)_{v_{\phi}}$ in such a way that our model is able to reproduce the main instability features deduced from PIC simulations. Based on this model with a kinetic non linearity for SRS, we have conducted numerical simulations that currently allow to describe plasmas of thousand wavelengths in length and several hundred of wavelengths in width. Similar work has, to our knowledge, yet only been done in configurations different from ours, namely 1) for EPWs without self-consistent SRScoupling, and in the frame co-moving with the EPW by Brunner $^{21}$, and 2) by Bénisti et al. ${ }^{43}$ in homogeneous plasmas, by comparing simulation results with kinetic simulations taken from the literature, extended later for spatial inhomogeneity ${ }^{44}$. Here, we directly compare 2D simulations from our 3 -wave model with 2D PIC simulations for the same, well-prepared plasma and laser beam conditions using the code EMI2D ${ }^{47,48}$. As discussed above, 2D effects such as side losses due to escaping trapped particles, diffusion effects in the phase and configuration space, may play a role but should not be too strong in our configuration. However, EPW front bowing (fluid simulation) / EPW bowing (PIC simulation) is expected to be important.

This article is organized as follows. In Sec. II we derive the three-wave enveloped equations in a weakly inhomogeneous plasma in the linear regime. Section III is devoted to the nonlinear frequency shift derivation and the nonlinear Landau damping expression. Comparison and benchmarks with 2D PIC simulations are presented in Sec. IV. The results are discussed in Sec. V.

\section{MODEL}

\section{A. Three-wave enveloped equations}

In the following the laser pump wave ('0') and the Langmuir wave ('L') propagate in positive direction along the $z$ axis, the Raman backscattered light wave ('R') propagates in opposite direction. Thus, $E_{0}$ and $E_{R}$ denote the complex envelopes of the pump and backscattered electromagnetic waves and $\tilde{E}_{L}$ is the complex electric field associated with the electron plasma wave. Let $\omega_{0}, \omega_{R}, k_{0}$ and $k_{R}$ having fixed values and verifying the dispersion relation: $\omega_{\alpha}^{2}=\omega_{p e}^{2}+k_{\alpha} c_{0}^{2}$, with $\alpha=\{0, R\}$, $\omega_{p e}$ the plasma frequency and $c_{0}$ the speed of light in vacuum. The EPW frequency $\omega_{L}$ and wave vector $k_{L}$ have fixed values and verify the plasma wave dispersion relation: $\omega_{L}^{2}=\omega_{p e}^{2}+3 v_{\mathrm{th}}^{2} k_{L}^{2}$, where $v_{\text {th }}$ is the electron thermal velocity.

Using the Slowly Varying Envelope Approximation (SEVA) and paraxial approximations, the energy exchange between these three waves in a stationary, underdense and isothermal plasma, is described by the fol- 
lowing well-known system of equations ${ }^{6}$ :

$$
\begin{aligned}
\mathcal{L}_{0} E_{0} & =-\frac{e k_{L}}{4 m_{e}} \frac{1}{\omega_{R}} E_{L} E_{R} e^{i \Psi}, \\
\mathcal{L}_{1} E_{R} & =\frac{e k_{L}}{4 m_{e}} \frac{1}{\omega_{0}} E_{0} \tilde{E}_{L}^{*} e^{-i \Psi}, \\
\mathcal{L}_{2} \tilde{E}_{L} & =\frac{e k_{L}}{4 m_{e}} \frac{\omega_{p e}^{2}}{\omega_{0} \omega_{L} \omega_{R}} E_{0} E_{R}^{*} e^{-i \Psi},
\end{aligned}
$$

where $e$ is the electron charge, $m_{e}$ the electron mass:

$$
\begin{aligned}
\mathcal{L}_{0} & =\left(\frac{\partial}{\partial t}+\nu_{0}+v_{g 0} \frac{\partial}{\partial z}+\frac{1}{2} \frac{\partial v_{g 0}}{\partial z}-i \frac{c_{0}^{2}}{2 \omega_{0}} \nabla_{\perp}^{2}\right) \\
\mathcal{L}_{1} & =\left(\frac{\partial}{\partial t}+\nu_{R}+v_{g R} \frac{\partial}{\partial z}+\frac{1}{2} \frac{\partial v_{g R}}{\partial z}-i \frac{c_{0}^{2}}{2 \omega_{R}} \nabla_{\perp}^{2}\right) \\
\mathcal{L}_{2} & =\left(\frac{\partial}{\partial t}+\nu_{L}+\nu_{\text {coll }}+v_{g L} \frac{\partial}{\partial z}+\frac{1}{2} \frac{\partial v_{g L}}{\partial z}-i \frac{v_{g L}}{2 k_{L}} \nabla_{\perp}^{2}\right) .
\end{aligned}
$$

The group velocities of these waves are denoted as $v_{g 0}$, $v_{g L}(>0)$, and $v_{g R}(<0)$, and their damping coefficients as $\nu_{0}, \nu_{R}$, and $\nu_{L}$. In inhomogeneous plasmas, the phase $\Psi$ appearing in Eqs. (1) for the complex wave envelopes, is due to the mismatch in the resonance conditions, $\Delta k=$ $k_{0}-k_{R}-k_{L}$ and $\Delta \omega=\omega_{0}-\omega_{R}-\omega_{L}$, and is defined by

$$
\Psi=-\Delta \omega t+\int_{z_{\mathrm{ref}}}^{z} \Delta k\left(z^{\prime}\right) \mathrm{d} z^{\prime}
$$

where $z_{\text {ref }}$ is an arbitrary reference position where the resonance conditions are satisfied. As mentioned earlier and in contrast to other work, e.g. Bénisti ${ }^{44}$, our model equations have been derived in applying consequently the linear dispersion relations, which is adequate in the context of driven plasma waves. For this reason, the non linear wave features will occur solely in the wave amplitudes. The damping coefficients in the set of Eqs. (1) account for electromagnetic waves' collisional absorption, $\nu_{0, R}$, while Langmuir wave's damping term holds for collisions $\nu_{\text {coll }}$ and Landau damping $\nu_{L}$.

\section{B. Coupling in a weakly inhomogeneous plasma}

We consider a weakly inhomogeneous plasma along $z$, with density $n_{e}(z)$, and we define a reference point $z_{\text {ref }}$, such that $n_{e}\left(z_{\text {ref }}\right)=N_{\text {ref }}$, where $N_{\text {ref }}$ is a given reference density around which the density profile is developed. Consequently the plasma frequency and the dispersion relations can be developed around this point,

$$
\omega_{p e}^{2}\left(n_{e}\right)=\frac{e^{2} N_{\mathrm{ref}}}{m_{e} \varepsilon_{0}}+\frac{e^{2}}{m_{e} \varepsilon_{0}}\left(n_{e}-N_{\mathrm{ref}}\right),
$$

By introducing the coupling coefficients:

$$
\Gamma_{0}=-\frac{e k_{L}}{4 m_{e}} \frac{1}{\omega_{R}}, \Gamma_{R}=\frac{e k_{L}}{4 m_{e}} \frac{1}{\omega_{0}}, \Gamma_{L}=\frac{e k_{L}}{4 m_{e}} \frac{\omega_{p e}^{2}}{\omega_{0} \omega_{L} \omega_{R}}
$$

and the substitution $E_{L}=\tilde{E}_{L} e^{i \Psi}$, one obtains the following set of equations for the 3-wave coupling,

$$
\begin{aligned}
\mathcal{L}_{0} E_{0} & =\Gamma_{0} E_{L} E_{R} \\
\mathcal{L}_{1} E_{R} & =\Gamma_{R} E_{0} E_{L}^{*} \\
\left(\mathcal{L}_{2}+i \frac{\left(n_{e}-N_{\mathrm{ref}}\right) e^{2}}{2 \omega_{L} m_{e} \varepsilon_{0}}\right) E_{L} & =\Gamma_{L} E_{0} E_{R}^{*} .
\end{aligned}
$$

As worked out by Rosenbluth ${ }^{49}$, solutions of the previous system depends on the density profile, via the $i\left(e^{2} / 2 \omega_{L} m_{e} \varepsilon_{0}\right)\left[n_{e}(z)-N_{\text {ref }}\right]$ term. For a linear density profile, i.e. $\left[n_{e}(z)-N_{\text {ref }}\right] \propto \kappa^{\prime} z$, with $\kappa^{\prime} \equiv$ $\partial_{z} \Delta k(z)=\left(6 k_{L} \lambda_{D e}^{2} L_{\nabla}\right)^{-1}$ with $L_{\nabla}=N_{\text {ref }}\left(\partial_{z} n_{e}\right)^{-1}$, both the backscattered light and the Langmuir waves are amplified in a limited zone in between two points, called "turning points", from either side of the resonance point with an amplification $\propto \exp G_{R}$, where $G_{R}$ is the gain denoted here as 'Rosenbluth gain' and given by

$$
G_{R}=\pi \Gamma_{R} \Gamma_{L} E_{0}^{2} /\left|\kappa^{\prime} v_{g R} v_{g L}\right|,
$$

or, in a more practical expression:

$$
G_{R}=0.9 \cdot 10^{-3} I_{15} L_{\nabla} k_{L}^{2} / k_{R},
$$

where $I_{15}$ is the intensity expressed in multiple of $10^{15}$ $\mathrm{W} / \mathrm{cm}^{2}$.

\section{MODELLING OF THE NON LINEAR LANGMUIR WAVE}

The equation for the SRS-driven Langmuir wave envelope as used in Eq. (4c), does not account for non linear effects arising during its propagation. In the following we develop a fluid-type model for Langmuir waves that takes into account the fact that trapped electrons are generated when the Langmuir wave amplitude grows. Our model relies on previously developed models by other authors $^{10,12,29}$, partially for the case of stimulated Brillouin scattering ${ }^{50,51}$ off ion acoustic waves.

When EPW grows in amplitude, the population of electron trapped in the potential wells increases leading to a modification of the dispersion relation. This increase results in a flattening of the electron distribution function around the phase velocity mainly due to the effect of electron trapping in the EPW. Thus, two important nonlinear consequences arise: i) the modification (decrease) of the electron plasma wave frequency and ii) the decrease or even the cancellation of Landau damping. Primarily these effects are one-dimensional, but collisions and gradients in the fields in the longitudinal and transverse dimension can lead to escaping and detrapping of particles out of the region of resonant wave-wave interaction. We discuss in the following those two features of our model: the non linear frequency shift as a possible mechanism of saturation due to a loss of resonance and a possible mechanism of autoresonace in an inhomogeneous plasma, and the non linear Landau damping that is responsible of kinetic inflation. 


\section{A. Non linear frequency shift}

The following modelling for the frequency shift induced by electron trapping is adapted from a model developed by Divol and Williams ${ }^{50,51}$ for the case of stimulated Brillouin scattering in 1D homogeneous plasma. They suggest a parametrized expansion of the particle distribution function $f(v)$ up to the second order derivative in velocity around the plasma wave phase velocity, $v_{\varphi}$, introducing the parameter $\delta$. This parameter accounts for the width of the plateau created by trapping effects. The parametrized distribution reads:

$$
f(v)=f_{0}(v)+\beta_{1} f_{1}(v)+\beta_{2} f_{2}(v)
$$

where $f_{0}(v)=\exp \left\{-v^{2} /\left(2 v_{\text {th }}\right)\right\} /\left[(2 \pi)^{1 / 2} v_{\text {th }}\right]$ stands for the unperturbed Maxwellian distribution function; the terms $\beta_{1} f_{1}(v)$ and $\beta_{2} f_{2}(v)$ are a development following the argument of Ref. ${ }^{12}$ in separating the non linear part from the linear contribution to the dispersion, via $\left.\varepsilon(k, \omega) \equiv \varepsilon(k, \omega)\right|_{\omega=\omega_{L}}+\left.(\partial \varepsilon / \partial \omega)\right|_{\omega=\omega_{L}} \delta \omega_{\mathrm{NL}}$ in Poisson's equation for $\delta f_{\mathrm{NL}} \equiv \beta_{1} f_{1}(v)+\beta_{2} f_{2}(v)$,

$$
i k\left(\frac{\partial \varepsilon}{\partial \omega}\right)_{\omega_{L}} \delta \omega^{\mathrm{NL}} E_{L}(k)=-\frac{N_{0} e}{\varepsilon_{0}} \int_{-\infty}^{\infty} \delta f_{\mathrm{NL}}(k, v) d v .
$$

As introduced before, Morales and O'Neil's expansion $^{12}$ obtained in the sudden case, up to second order, of $\delta f_{\mathrm{NL}} \equiv f_{0}(v+\Delta v)-f_{0}(v)$ yields to a frequency shift $\delta \omega_{\mathrm{M}}^{\mathrm{NL}}$ proportional to the bounce frequency $\omega_{B}$ :

$$
\begin{aligned}
\delta \omega_{\mathrm{M}}^{\mathrm{NL}} & \simeq-1.63 \frac{\omega_{B}}{k_{L}} v_{\phi}^{2}\left(\frac{\partial^{2} f_{0}}{\partial v^{2}}\right)_{v_{\phi}}\left(\frac{\partial \varepsilon}{\partial \omega}\right)_{\omega_{L}}^{-1} \\
& =-\eta_{\mathrm{M}} \omega_{L}\left|\frac{\delta n}{N_{0}}\right|^{1 / 2}
\end{aligned}
$$

i.e. being proportional to square root of the electrostatic field, $\omega_{B}=\left|e E_{L} k_{L} / m_{e}\right|^{1 / 2}$ or, equivalently, to the Langmuir wave density perturbation, $\omega_{B}=\omega_{L}\left|\delta n / N_{0}\right|^{1 / 2}$, with $\eta_{\mathrm{M}}=0.823 v_{\phi}^{3}\left(\partial^{2} f_{0} / \partial v^{2}\right) v_{\phi}$.

The ansatz by Divol and Williams ${ }^{50,51}$ follows this expansion by considering that the distribution function has a plateau around the phase velocity, with a width $\delta \equiv\left|v-v_{\phi}\right|$, such that $f_{1}(v) \equiv v \exp \left\{-v^{2} /\left(2 \delta^{2}\right)\right\}$ and $f_{2}(v) \equiv \zeta\left(v^{2}-\delta^{2}\right) \delta \exp \left\{-v^{2} /\left(2 \delta^{2}\right)\right\}$. The parameters in Eq. (7) are the 1 st and 2 nd order derivates of $f_{0}$, namely $\beta_{1}=-\left(\partial f_{0} / \partial v\right)_{v_{\phi}}$ and $\beta_{2}=-\left(\partial^{2} f_{0} / \partial v^{2}\right) v_{\phi}$. From the dispersion relation Eq. (8) only the second order term contributes to the integral so that, with $\left.(\partial \varepsilon / \partial \omega)\right|_{\omega=\omega_{L}} \equiv 2 / \omega_{L}$, the non linear frequency shift $\delta \omega^{\mathrm{NL}}$ results in

$$
\frac{\delta \omega^{\mathrm{NL}}}{\omega_{L}}=-8 \zeta \sqrt{2 \pi} v_{\phi}\left(\frac{\partial^{2} f_{0}}{\partial v^{2}}\right)_{v_{\phi}} \delta \equiv-\eta \frac{\delta}{v_{\phi}} .
$$

The comparison with Morales/O'Neil's expression, Eq. (9), gives a direct relation between the width, $\delta$, in the plateau of $f$ and the electrostatic field $E_{L}$,

$$
\delta \simeq \frac{1.63}{16 \zeta \sqrt{2 \pi}}\left|\frac{e E_{L}}{m_{e} k_{L}}\right|^{1 / 2} v_{\phi}=0.04 \zeta^{-1} \frac{\omega_{B}}{k_{L}} v_{\phi}
$$

To get equivalence between $\eta \delta / v_{\phi} \equiv \eta_{M}\left|\delta n / N_{0}\right|^{1 / 2}$, the value of $\zeta$ has to be $\zeta=0.04$. From this ansatz, the kinetic energy related to trapped particles can be computed from both $f_{1}$ and $f_{2}$

$$
\mathcal{E}=\mathcal{E}_{1}+\mathcal{E}_{2}
$$

yielding

$$
\begin{aligned}
& \mathcal{E}_{1}=\sqrt{2 \pi} m_{e} n_{e}\left(\frac{\partial f_{0}}{\partial v}\right)_{v_{\phi}} v_{\phi} \delta^{3} \\
& \mathcal{E}_{2}=\zeta \sqrt{2 \pi} m_{e} n_{e}\left(\frac{\partial^{2} f_{0}}{\partial v^{2}}\right)_{v_{\phi}} \delta^{5} .
\end{aligned}
$$

For

$$
\zeta\left|\frac{\partial^{2} f_{0}}{\partial v^{2}}\right|_{v_{\phi}} \delta^{2} \ll\left|\frac{\partial f_{0}}{\partial v}\right|_{v_{\phi}} v_{\phi}
$$

which is usually the case, the second term $\mathcal{E}_{2}$ plays a negligible role in the energy variation in time, so that it is well approximated by

$$
\frac{\mathrm{d} \mathcal{E}}{\mathrm{d} t} \simeq \frac{\mathrm{d} \mathcal{E}_{1}}{\mathrm{~d} t}=\sqrt{\frac{8}{\pi}} \nu_{L, 0} \frac{m_{e} n_{e}^{2}}{\omega_{L} v_{\phi}} \frac{\mathrm{d} \delta^{3}}{\mathrm{~d} t}
$$

in which the linear Landau damping coefficient, $\nu_{L, 0}$ evaluated for the initial distribution $f_{e}(v) \equiv f_{0}(v)$

$$
\nu_{L, 0}=\left.\frac{\pi}{2} \omega_{L} \frac{\omega_{p e}^{2}}{k_{L}^{2}} \frac{1}{n_{e}} \frac{\partial f_{0}}{\partial v}\right|_{v=v_{\phi}}
$$

occurs. Comparing this to the the local electrostatic energy for the plasma wave, given by

$$
W_{\mathrm{EPW}}=\frac{\varepsilon_{0}}{4}\left(\frac{\partial \omega \epsilon}{\partial \omega}\right)_{\omega_{L}}\left|E_{L}\right|^{2}=\frac{1}{2} m_{e} n_{e}^{2} \frac{\omega_{B}^{4}}{\omega_{p e}^{2} k_{L}^{2}},
$$

where $\epsilon$ is the dielectric function and $\varepsilon_{0}$ the vacuum permittivity, one can determine an effective Landau damping coefficient, $\nu_{L, \text { eff }}$ via the relation

$$
\frac{d \mathcal{E}_{1}}{d t} \simeq 2 \nu_{L, \mathrm{eff}} W_{\mathrm{EPW}}
$$

This yields a differential equation for $\delta^{3}$

$$
\frac{\mathrm{d} \delta^{3}}{\mathrm{~d} t}=\frac{\nu_{L, \mathrm{eff}}}{\nu_{L, 0}} \sqrt{\frac{\pi}{8}} \omega_{B} v_{\mathrm{tr}}^{3}
$$

where the auxiliary velocity $v_{\mathrm{tr}}=\omega_{B} / k_{L}$ has been introduced, that is related to the width of the plateau in the distribution function around $v_{\phi}$ due to electron trapping. 
In Refs. 50 and 51, a set of differential equations has been proposed to describe adequately the evolution of the quantity $\delta$ that determines the frequency shift in the equation of the EPW, namely

$$
\begin{aligned}
& \frac{\partial \delta^{3}}{\partial t}=\mathcal{H}\left(\delta<\alpha v_{\mathrm{tr}}\right) \sqrt{\frac{\pi}{8}} \omega_{B} v_{\mathrm{tr}}^{3}, \\
& \delta=\operatorname{Max}\left(0,\left(\delta^{3}\right)^{\frac{1}{3}}\right), \\
& \left(\frac{\partial}{\partial t}+v_{\phi} \frac{\partial}{\partial z}\right) \delta=0 .
\end{aligned}
$$

Equation (20a) uses Eq. (19) under the hypothesis that the effective Landau damping is equivalent to the linear Landau damping, $\nu_{L, \text { eff }} \equiv \nu_{L, 0}$ as long as the width of the plateau is limited ${ }^{25}$, i.e. less than $\alpha v_{\text {tr }}$, where $\alpha$ is a numerical factor of the order of unity (In practice, the value of $\alpha=2$ is recommended based on simulations with kinetic $\left.\operatorname{codes}^{25}\right)$. Once the plateau width $\delta$ reaches or exceeds $\alpha v_{\text {tr }}$, the Landau damping coefficient should become negligible, so that in the model the growth of the plateau width is interrupted. This cancellation is taken into account via the Heaviside function $\mathcal{H}$. The other two equations in Eqs.(20) describe respectively the positivity of the plateau width, and its propagation at the phase velocity due to trapped particles. Divol ${ }^{50}$ proposes also a diffusion term in Eq. (20c) to account for side losses. Work with S. Brunner leads alternatively to a group approach for side losses ${ }^{52}$.

\section{B. EPW damping}

The different sources of expected damping are electronion collisions and inverse Bremsstrahlung. Given the plasma parameters, we can estimate their respective frequencies to $\nu_{e i} / \omega_{p e} \sim 0.16 \%$ and $\nu_{B} / \omega_{p e} \sim 0.04 \%$. In addition, and as discussed above, as long as side or collisional losses can be neglected, the Landau damping is the dominant damping effects. In the following, we focus on the best modelling of this effect but as mentioned in Part A, collisionnal damping may easily be taken into account via the $\nu_{\text {coll }}$ coefficient.

Following Eqs.(20), the model for the non linear frequency shift, the effective Landau damping rate should abruptly vanish when $\delta$ reaches the value defined by $\alpha v_{\text {tr }}$,

$$
\frac{\nu_{\mathrm{L}, \mathrm{eff}}}{\nu_{L, 0}}=\mathcal{H}\left(\delta<\alpha v_{\mathrm{tr}}\right),
$$

acting like a cut off. This method was used in Refs. 50 and 51 for the ion Landau damping contribution, $\nu_{L, i}$ to the total damping of ion acoustic waves, $\nu_{\text {IAW }}$. This sharp cut off of Landau damping is a drawback for numerical schemes and may cause numerical instability in multi-dimensional simulations.

Alternative methods were investigated by different authors for evaluating the evolution of the Langmuir damping rate due to the increasing population of trapped electrons. In Ref 30 the Landau damping rate $\nu_{L \text {,eff }}(t)$ follows the differential equation

$$
\frac{\partial}{\partial t}\left[\left(\nu_{L, 0}-\nu_{L, \mathrm{eff}}(t)\right) \omega_{B}^{3}(t)\right]=\kappa \nu_{L, \mathrm{eff}}(t) \omega_{B}^{4}(t)
$$

where $\nu_{L, 0}$ stands for the linear Landau damping, $\omega_{B}=$ $\omega_{p e}\left|\delta n / N_{0}\right|^{1 / 2}$ the bounce frequency, $\delta n$ the plasma wave amplitude, and $\kappa$ a free parameter calibrated with kinetic simulations.

This expression of the Landau damping rate is convenient for numerical simulations as it expresses the damping value as a function of the history of the bounce frequency $\omega_{B}$ (that could be recorded at each time step).

However, as discussed in Ref.53 this description is limited to an EPW monotonically growing in amplitude. Indeed, we numerically verify that when the plasma wave stops growing, this expression can lead to non physical solutions such as negative values or the continuous growth of the Landau damping rate $\nu_{L \text {,eff }}$ which cannot be correct because the physics that would allow such evolution (detrapping, non linear evolution of the group velocity) was not taken into account.

Another expression for the effective Landau damping rate, used in Refs 20, 31, and 32 allows for gradual decrease of the damping rate together with the increase of the trapped particle population via an integral over the local bounce frequency, namely

$$
\frac{\nu_{L, \mathrm{eff}}}{\nu_{L, 0}}=\frac{1}{1+\frac{1}{2 \pi} \int^{t} \omega_{B}\left(t^{\prime}\right) \mathrm{d} t^{\prime}} .
$$

Though this last expression is very computationally convenient, it does not allow the landau damping to restart. In addition, neither of these models for Landau damping is consistent with the formulation of the non linear frequency shift derived here, as these two terms should both depend on the $\delta$ quantity. Thus, we propose here a new expression for the non linear Landau damping which explicitly depends on $\delta$ :

$$
\frac{\nu_{L, \mathrm{eff}}}{\nu_{L, 0}}=\frac{1}{1+\left(\frac{\beta \delta}{v_{\mathrm{tr}}}\right)^{4}}
$$

where $\beta$ is a numerical parameter whose value will be determined later (in section IV B) by comparing the value of the growth rate measured in the fluid simulations to the one observed in PIC simulations.

We have examined how these models compare to each other when the Langmuir wave grows in the limits discussed in Refs. 30 and 53. Thus, we consider an EPW driven by an exponentially growing driver. Eq. (21) evidently leads to an abrupt jump to zero at a time instant around which the expressions following Eqs. (22), (23) and (24) smoothly decay also to zero, in a similar manner, as shown in Fig. 1. Except the Heaviside treatment of Landau damping, all the models discussed here present a similar behavior. Eqs. (22) and (23) were calibrated with 


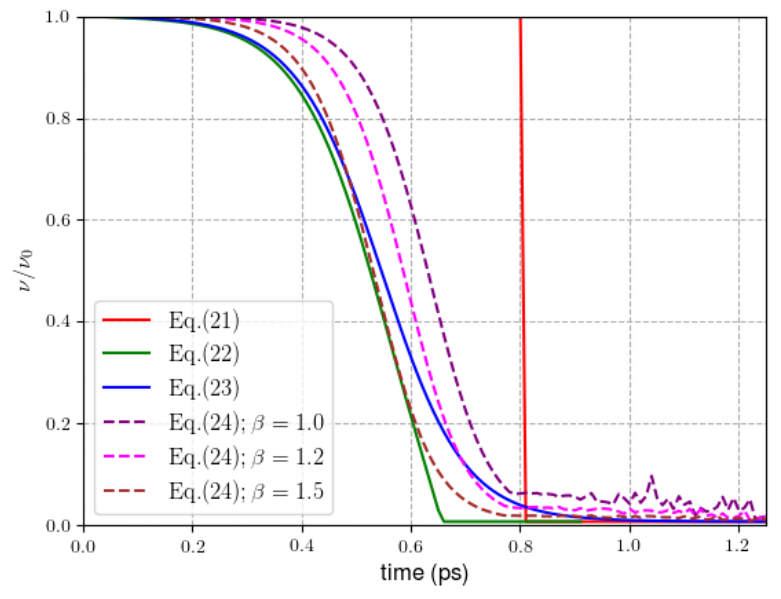

Figure 1. Four different models of nonlinear Landau damping, Eqs.(21) (red curve - Heaviside), (22) (green curve), (23) (blue curve), and Eq.(24) (dotted curves) for different values of the $\beta$ parameter, for a EPW under the influence of an exponentially growing driver applied to Eq.(1c).

homogeneous 1D PIC simulations ${ }^{20,30}$. The free parameter $\beta$ in our Landau damping formulation (24) is equally calibrated with a 2D homogeneous PIC simulation as we shall see later.

\section{Full model}

Finally, we propose the following full set of equations:

$$
\begin{aligned}
\mathcal{L}_{0} E_{0} & =\Gamma_{0} E_{L} E_{R}(25 \mathrm{a}) \\
\mathcal{L}_{1} E_{R} & =\Gamma_{R} E_{0} E_{L}^{*}(25 \mathrm{~b}) \\
\left(\mathcal{L}_{2}+i \frac{\left(n_{e}-N_{0}\right) e^{2}}{2 \omega_{L} m_{e} \varepsilon_{0}}-i \delta \omega\right) E_{L} & =\Gamma_{L} E_{0} E_{R}^{*}(25 \mathrm{c})
\end{aligned}
$$

in which $\delta \omega=\left|\delta \omega^{\mathrm{NL}}\right|=\eta\left(\omega_{L} / v_{\phi}\right) \times \delta$ from Eq. (10) is evaluated using Eqs. (20a)-(20c), and where $\mathcal{L}_{2}$ contains the time dependent Landau damping coefficient

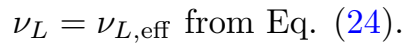

In a homogeneous plasma, the non linear frequency shift detunes the three waves coupling, preventing the exponential growth of the EPW. This saturation mechanism occurs long before the depletion of the pump wave.

In the opposite case as the frequency shift due to electron trapping is always negative, it could actually compensate the phase mismatch due to the electron density inhomogeneity. Consequently, in a positive linear density gradient, the nonlinear frequency shift allows the growth of the plasma wave far beyond the Rosenbluth gain. This so called autoresonance phenomena in one dimensional plasma was studied by Yaakobi ${ }^{36}$ and Chapman ${ }^{31,32}$.
The next section is dedicated to the validation of our full model by comparison with 2D PIC simulations in well controlled situations.

\section{COMPARISONS WITH PIC SIMULATIONS}

\section{A. Simulation methodology}

We compare now the results obtained with SIERA, our new paraxial code solving the full set of equations (25a) - (25c), (24), and (10), and Emi2D ${ }^{47,48}$, a Maxwell-PIC code. We consider a $100 \mu \mathrm{m}$ long and $54 \mu \mathrm{m}$ large plasma with a linear electron density profile along the longitudinal z-axis. We use the gradient length $L_{\nabla}$ in order to characterize the plasma inhomogeneity

$$
n_{e}(z)=N_{0}\left(1+\frac{z-z_{\mathrm{ref}}}{L_{\nabla}}\right)
$$

and the reference position is set at the center of the simulation box with the value $N_{0}=0.05 n_{c}$. A homogeneous plasma corresponds to the infinite gradient length case. For the sake of clarity and concision, we only present here 2 cases : a homogeneous and a inhomogeneous configurations. Few other cases were studied ${ }^{46}$.

Typical electron densities span from $\sim 2 \%$ to $\sim 8 \%$ over $100 \mu \mathrm{m}$ corresponding to $L_{\nabla}=75.75 \mu \mathrm{m}$, see Fig. 2. The temperature is kept constant at $1 \mathrm{keV}$ so the $k_{L} \lambda_{D}$ parameter equals 0.34 at the center of the box. The pump wave propagates from the left to the right, has a gaussian shape with a numerical aperture $f_{\#}=16$ and a $0.351 \mu \mathrm{m}$ wavelength corresponding to the third harmonic $(3 \omega)$ of a Nd-glass laser. The peak intensity is set to $I_{0}=5 \cdot 10^{15} \mathrm{~W} \cdot \mathrm{cm}^{-2}$ at the center of the simulation box. In order to compare kinetic and fluid simulation results, we need to control perfectly when and where the Raman scattering will occur and grow, and to gloss over the natural PIC noise. To do this, the instability is triggered with a counter propagating and monochromatic seed such as the matching conditions are verified at the reference density $N_{0}$. This seed is a plane wave, whose intensity is $I_{\text {seed }}=2 \cdot 10^{-3} I_{0}$ which is willingly high in order to make the instability rise quickly in the PIC simulation, saving computational time. The PIC simulations were performed on 2,048 IBM Bluegene Q CPU with 100 particles per cell and the grid was 20,000 $\times 10,000$ cells corresponding to $d x=d y=0.1 k_{0}^{-1}$.

For all simulations the pump wave is initialized with a Gaussian beam boundary condition at the left side, and the right side is an outgoing boundary condition. The seed is initialized with a plane wave boundary condition at the right side. The left side is an outgoing boundary condition. In the fluid simulation, the EPW is the result of the EM waves coupling and has an outgoing boundary condition at the right side. In the PIC simulations, the ions are motionless, which is a valid hypothesis considering the time scale studied here. The transverse boundary 


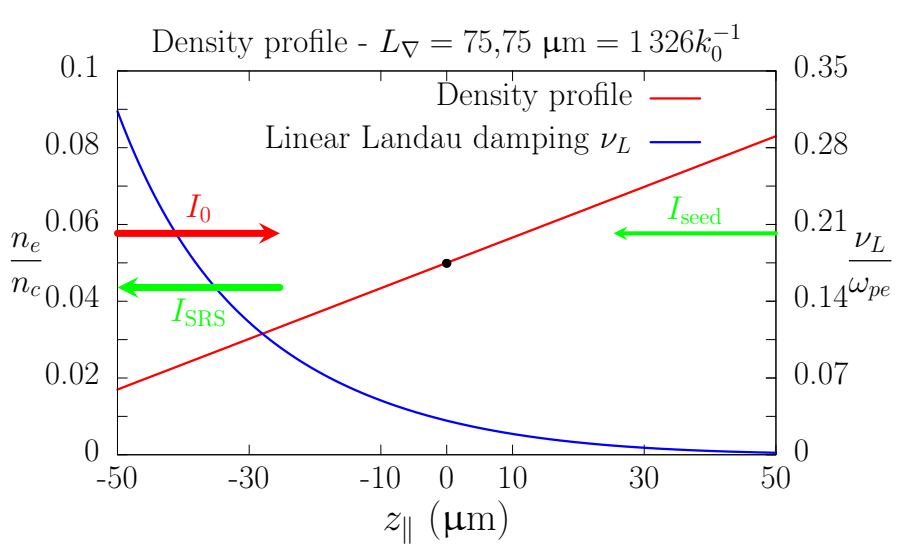

Figure 2. Electron density profile (red curve) and linear Landau damping (blue curve) along the z-axis in the simulation box for $L_{\nabla}=75.8 \mu \mathrm{m}$. The reference position, where the matching conditions are imposed, is represented by a black bullet. The laser propagates from the left to the right.

condition is periodic but the system is large enough to be unsignificant. In the longitudinal direction, there is a void area between the end of the plasma and the end of the simulation domain: when the electrons arrive in this zone, an ambipolar electric field is created because of the charge separation, which leads to a return of the particles with an opposite velocity. If they left the simulation domain they would be reflected to mimic this ambipolar field. The PIC simulation is stopped before these particles pollute the areas of interest.

The use of a linear density profile allows us to perfectly control the wave coupling in the sense that the Rosenbluth amplification depends solely of the profile slope (Cf. Eq. (6)). For this case we obtain the low Rosenbluth intensity gain $G_{R}=1.9$, preventing the instability saturation by pump depletion.

For a homogeneous plasma $\left(L_{\nabla} \rightarrow \infty\right)$, Raman backscattering will initially show an exponential growth in time. The SRS growth rate is given by:

$$
\gamma=-\frac{\nu_{L, \mathrm{eff}}}{2}+\gamma_{0} \sqrt{1+\left(\frac{-\nu_{L, \mathrm{eff}}}{2 \gamma_{0}}\right)^{2}}
$$

where $\gamma_{0}$ reads

$$
\gamma_{0}=\frac{1}{4} k_{L} \frac{\omega_{p e}}{\omega_{R} \omega_{L}}\left(\frac{e E_{0}}{m_{e} \omega_{0}}\right)
$$

is the linear growth rate, and $\nu_{L, \text { eff }}$ the effective (Landau) damping.

For the parameters described above, $\gamma_{0} \sim 2.4 \cdot 10^{13} \mathrm{~s}^{-1}$ and the linear Landau damping for $n_{e} / n_{c}=0.05$ is $\sim 0.03 \omega_{p e} \sim 3.6 \cdot 10^{13} \mathrm{~s}^{-1}$. In addition, without the kinetic effects, SRS saturation because of the pump depletion is given by the classical Manley-Rowe relation

$$
\frac{I_{\mathrm{SRS}}}{I_{0}}=-\frac{\omega_{R} v_{g 0}}{\omega_{0} v_{g R}} \sim 0.79
$$

\section{B. The 2D homogenous case: calibration of $\beta$ parameter}

Homogeneous configurations are usually presented as textbook cases but are actually difficult to handle because the system easily reaches the stage of pump depletion, with complicated spatio-temporal dynamics. Fortunately, because the initial growth rate in this configuration depends on the effective Landau damping, see Eq. (26) the homogenous configuration should allow us to calibrate the $\beta$ parameter from (24). Indeed, the very first amplification, up to $0.5 \mathrm{ps}$, represents the growth of the SRS instability while the kinetic effects are developing. At this stage, the Landau damping has strongly decreased but the detuning of the whole system is not too strong, and the saturation due to the frequency shift is not reached yet. That is why the growth mechanism is similar to the linear one in the no damping limit.

Considering a 2D $5 \%$ critical homogeneous plasma with the simulation parameters previously described, we compare in Fig. 3 the results obtained with the PIC code and SIERA, for different values of $\beta$ parameter. The $\eta$ parameter is set to $\eta=0.07$. This value will be extensively discussed below, in the inhomogenous part. We recall that the value of $\eta$ affects the saturation mechanism but not the SRS growth rate (contrary to the $\beta$ coefficient), that is why the value of $\eta$ is not important here for the calibration of $\beta$.

As discussed above, the growth rate depends on the Landau damping via the $\beta$ coefficient. With $\beta=1$, its decrease is not fast enough and the EPW damping is too strong resulting in a too weak effective growth rate for the SRS instability. $\beta=1.5$, is not suitable for the opposite reason. From now, we choose $\beta=1.2$ as it appears to be the best value fitting the PIC simulation. Hence we find empirically a good agreement between PIC simulations and the wave coupling model for $\eta=0.07$ and $\beta \sim 1.2$ for an homogeneous plasma density profile. We will use the same parameters for an inhomogeneous density profile with a linear gradient, in the next section.

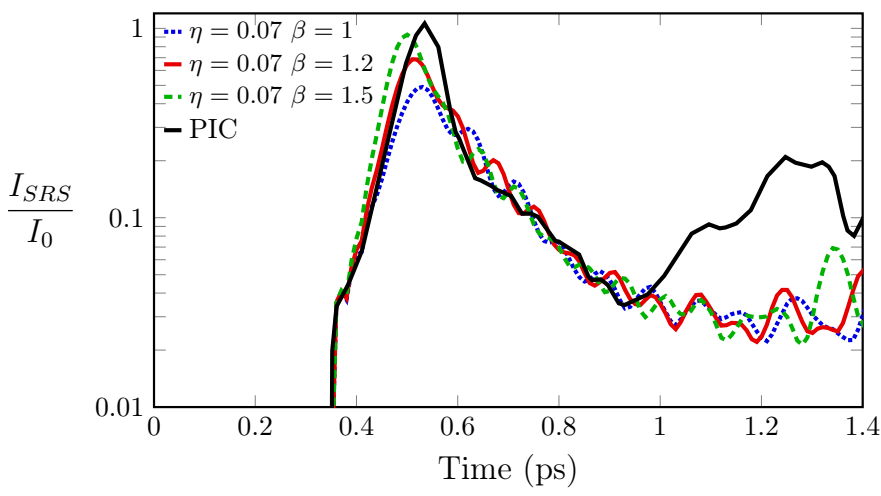

Figure 3. Comparison of the PIC reflectivity (black curve) and SIERA reflectivities in the homogeneous case for different values of $\beta$ parameter and $\eta=0.07: \beta=1$ (blue dotted), $\beta=1.2$ (red) and $\beta=1.5$ (green). 


\section{The 2D homogeneous case: kinetic effects}

The reflectivity, saturation and decrease - up to 0.95 ps - observed both in PIC and SIERA simulations are the consequences of the non linear frequency shift due to electron trapping as the EPW wave grows. This frequency shift detunes the system depriving it of an efficient coupling. The triggered instability is not sustained anymore resulting in the decrease of Raman reflectivity. In Fig. 4 the blue dotted line is the SIERA reflectivity when $\eta=0$ i.e. when the kinetic effects are not taken into account (and $\nu_{L}=0$ ). The SRS instability saturates because of the pump depletion and stabilizes at the theoretical level: $I_{\mathrm{SRS}} / I_{0}=0.79$.

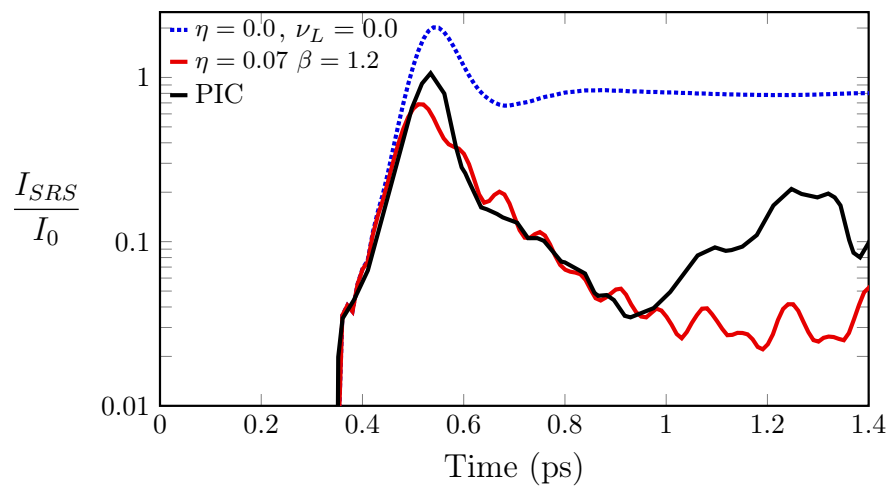

Figure 4. Comparison of temporal evolution of SRS reflectivity obtained with SIERA code without kinetic effects nor Landau damping (blue curve), with kinetic effects $(\eta=0.07$ and $\beta=1.2$ ) (red curve) and by PIC simulation (black curve).

The reduced formulation with the modeling of the kinetic effects and therefore the non linear frequency shift allow us to obtain and understand the first part of the simulation: the growth of SRS, its first saturation and decrease due to electron trapping. Despite this good agreement, we are not able to catch the second growth observed in the PIC simulation beyond 1 ps (Fig. 4).

This second growth is probably due to the emergence of frequencies initially contained in the natural noise inherent to a PIC code. Indeed, this broadband noise contains frequencies that could match the resonance conditions in the now detuned system. In order to investigate further, a method using Langevin equations in time and space for generating a broadband noise ${ }^{54}$ was implemented into SIERA. Preliminary results are very encouraging and confirm the great importance of noise terms for SRS in homogeneous cases, but a more detailed study is beyond the scope of this paper and still has to be done, regarding the noise in PIC simulations and the best way to simulate it in a fluid approach.

As the intensity of the counter-propagating trigger is far above the natural noise level of the PIC simulation, the system is not sensitive anymore to the noise in the linear inhomogeneous case previously described. Thus, this configuration is well controlled and allows us to isolate the non linear frequency shift due to kinetic effects solely. This inhomogenous case is discussed in the next section.

As SIERA computes the envelope of the different waves, we could compare their structures with the PIC simulation. The direct comparison between PIC and envelope codes is particularly difficult because of the numerous approximations of the latter. In order to make the comparison pertinent, one has to extract the envelope of the signal from the PIC simulations by means of Hilbert transforms, for example. This process is well described $^{21,22}$. Basically, an analytic signal $\left(a\left(z, x_{\perp}\right)\right)$ associated to the calculated data $\left(r\left(z, x_{\perp}\right)\right)$ is built so that $a\left(z, x_{\perp}\right)=r\left(z, x_{\perp}\right)+i \mathcal{H}[r]\left(z, x_{\perp}\right)=a_{m}\left(z, x_{\perp}\right) e^{i \phi\left(z, x_{\perp}\right)}$, where $\mathcal{H}$ is the so called Hilbert transform. In theses conditions, taking the modulus of the analytic signal $\left|a\left(z, x_{\perp}\right)\right|$ gives $a_{m}\left(z, x_{\perp}\right)$, the signal envelope. In Fig. 5 and Fig. 6 we compare the EPW envelope extracted from the PIC simulation (around the main SRS mode) to that from the fluid-like simulation at two different instants: just after the linear growth phase $(0.55 \mathrm{ps})$ and later (1.2 ps) when the system is completely detuned because of the non linear frequency shift. The global structure and spatial extension are well described by the fluid-like simulation and particularly the islets in the tail of the EPW. Unfortunately, because of the paraxial approximation, SIERA can not precisely compute the round structure of the EPW head (see Fig. 6).

\section{Results and analysis of the 2D inhomogenous PIC simulations}

In contrast to the case of a homogeneous plasma, presented above, the Raman scattering in the inhomogeneous profile chosen is governed by the resonance mismatch which results, in absence of kinetic effects, in spatial amplification of the seed signal. Pump depletion would only arise for high Rosenbluth gain values. With such initial and boundary conditions, the instability reaches quickly the linear state determined by Rosenbluth's theory, that is when kinetic effects are negligible, and then evolves softly toward the non linear kinetic regime as the EPW's amplitude increases. This is illustrated in Fig. 7. The time evolution of the PIC reflectivity is decomposed in three parts. The first, up to $0.7 \mathrm{ps}$, is associated to the Rosenbluth response of the system. The second, from 0.7 to $1.2 \mathrm{ps}$, is related to a destabilization due to kinetic effects. It is a transition phase. During this phase, the reflectivity is significantly higher that the one calculated using the Rosenbluth gain. Enhanced reflectivity due to kinetic effects is attributed to the auto resonance mechanism in which the induced frequency shift due to electron trapping enlarges the resonance region. To our knowledge, this is the first demonstration of auto resonance in a $2 \mathrm{D}$ geometry. The third phase exhibits a non stationary behaviour. This indicates that auto reso- 

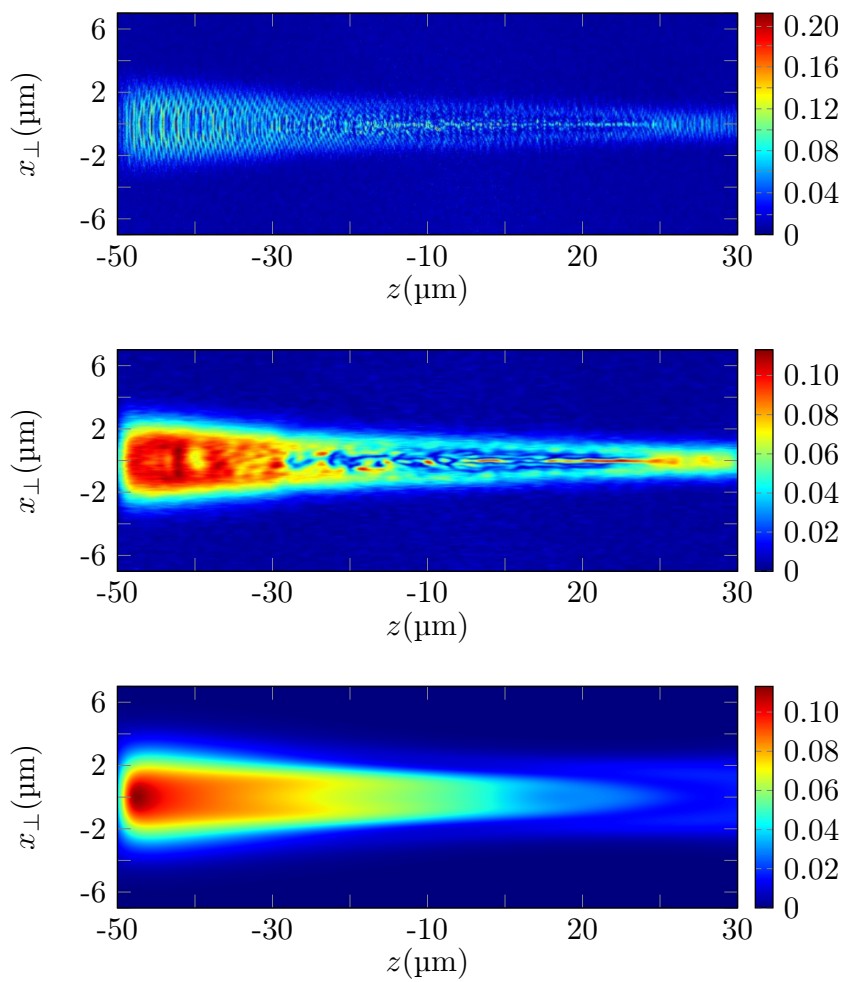

Figure 5. (Homogeneous plasma case) EPW field in $\left|\frac{\delta n_{e}}{N_{0}}\right|$ at $t=0.55$ ps. Top: Data given by the PIC code Emi2D. Middle: Envelope of the above signal, around the linear Raman mode, extracted from the PIC simulation with the Hilbert transform $^{13}$. The latter allows to identify the EPW amplitude out of the fast EPW oscillations as seen in the unfiltered signal (top). Bottom: Envelope of the EPW calculated with SIERA.

nance is not maintained because of geometrical evolution that competes with non linear effects. Modulations suggest that the SRS electric field may be decomposed into the following way: $E_{\mathrm{SRS}}=E_{\mathrm{Ros}}+E_{\mathrm{tr}} e^{-i \delta \omega t}+$ c.c. where $E_{\mathrm{Ros}}$ is the field associated to the Rosenbluth response and $E_{\mathrm{tr}}$ the field due to electron trapping.

Due to the low amplification level expected, the intensity of the reflected light should be composed of two major spectral contributions, shifted in frequency:

$$
I_{\mathrm{SRS}} \propto\left|E_{\mathrm{RoS}}\right|^{2}+\left|E_{\mathrm{tr}}\right|^{2}+E_{\mathrm{Ros}} E_{\mathrm{tr}} e^{-i \delta \omega t}+\text { c.c. }
$$

which is a signal oscillating at the frequency $\delta \omega$. This remark allows us to measure the frequency shift directly from the PIC reflectivity on Fig. 7, which is $(4.4 \pm 0.3) \cdot 10^{-2} \omega_{p e}$.

Now, we turn our attention to the PIC electron distribution function in a Raman active region of the system (Cf. Fig. 8), in order to estimate the width $\delta$ of the plateau due to electron trapping. This measure gives $\delta / c_{0} \sim 0.09 \pm 0.02$. Now, using Eq. (10) one can estimate
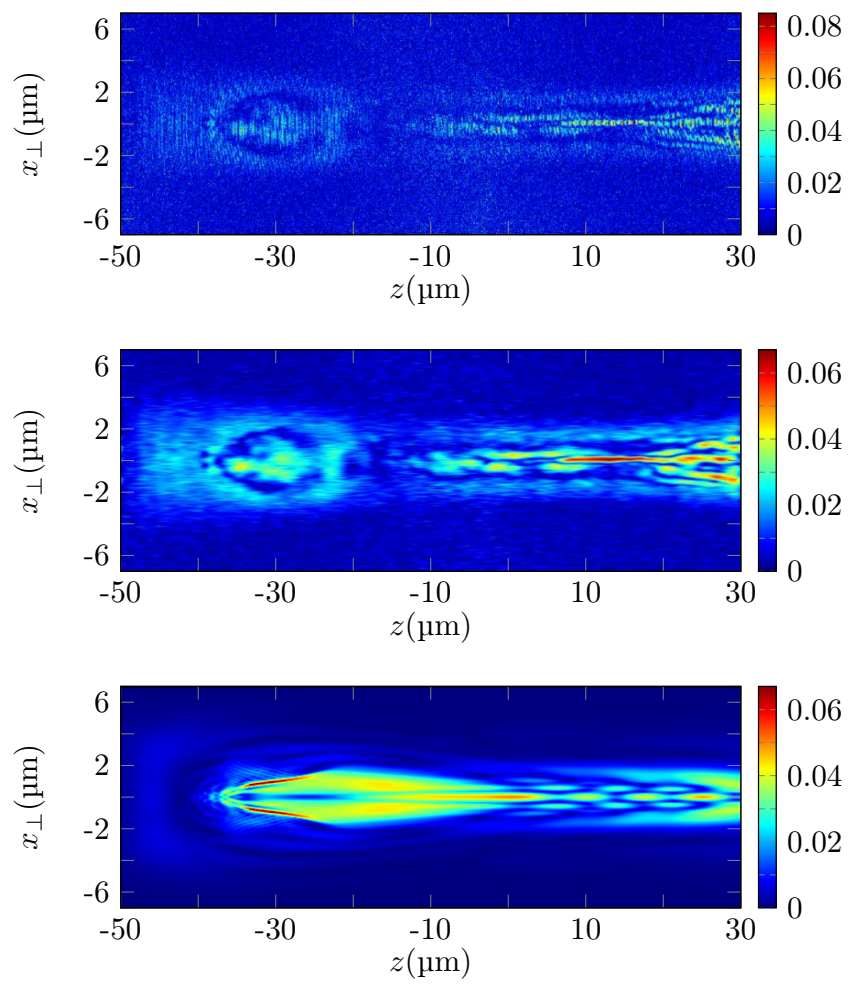

Figure 6. (Homogeneous plasma case) EPW field in $\left|\frac{\delta n_{e}}{N_{0}}\right|$ at $t=1.2$ ps. Top: Data given by the PIC code EmI2D. Middle: Envelope of the above signal, around the linear Raman mode, extracted with the Hilbert transform ${ }^{13}$. Bottom: Envelope of the EPW calculated with the fluid-like model in SIERA.

the value of $\eta$ given by the PIC simulation:

$$
\eta=\frac{\delta \omega}{\omega_{p e}} \frac{v_{\phi}}{\delta c_{0}} \approx 0.07( \pm 0.02) .
$$

This explains the choice of $\eta$ used in the homogeneous case as well.

\section{E. Comparisons and calibration of the reduced model}

The validity of the reduced model is assessed via the comparison of several quantities calculated in both fluidlike and PIC simulations. Moreover, for the purpose of establishing the model sensitivity to the $\eta$ parameter, several simulations of the same configuration, with different values for $\eta$, are required.

In Fig. 9 we present the instantaneous reflectivity given by the PIC code and SIERA, with $\eta=0.05,0.07$ and 0.09 where $\eta=0.07$ is supposed to be the centered value of our estimation. There is also a witness simulation with $\eta=0.00$, i.e. without kinetic effects, in order to settle the role of kinetic effects. In all cases, as expected in the first stage, the reflectivity reaches a level corresponding to the Rosenbluth amplification ${ }^{55}$, and stabilizes at 


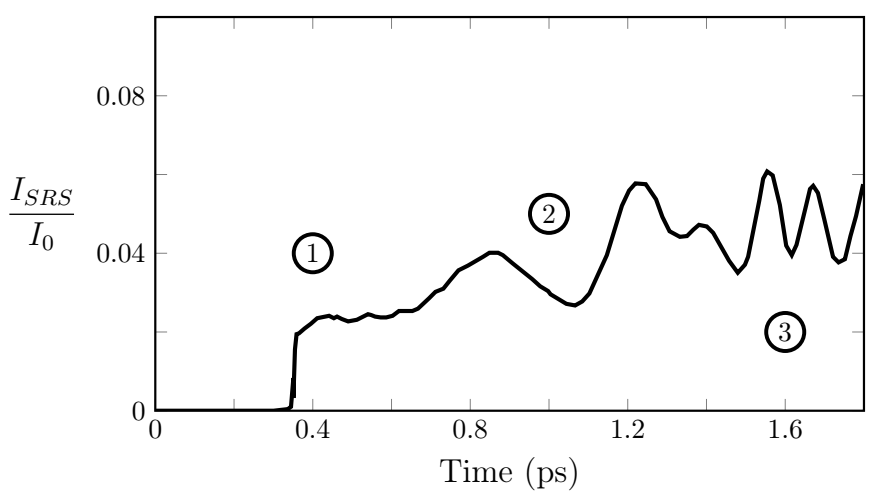

Figure 7. PIC instantaneous reflectivity versus time for the linear inhomogeneous case. The reflectivity presents three phases: (1) corresponds to the linear growth and Rosenbluth amplification; (3) exhibits some modulations due to the competition between geometric and kinetic effects; (2) is a transition phase.

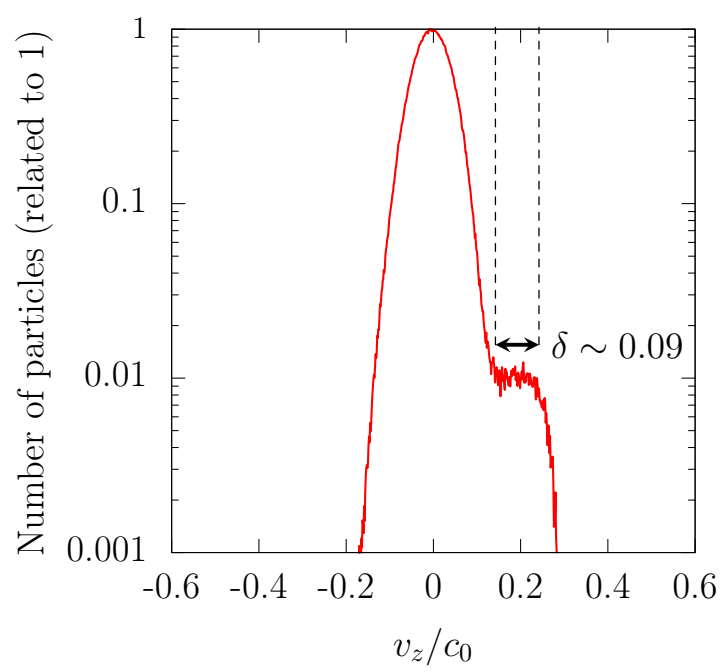

Figure 8. Reconstructed PIC distribution function in a Raman active area at $1.5 \mathrm{ps}$. The electron distribution function is spatially averaged around the resonant point over $2 \lambda_{L}$ in the propagation direction and $2 \lambda_{L}$ in the transverse direction.

a very precise value for the linear simulation. Simulations with $\eta>0$ produce more reflectivity compared to the linear case, indicating a destabilization of the system and the enlargement of the resonance area.

The parameter $\eta$ modifies the reflectivity history, and the best match with PIC calculation is for $\eta=0.07$ in agreement with the value estimated from the PIC code (28).

Now, we compare the z-position of the EPW amplitude first maximum versus time (Cf. Fig. 10). Indeed, these first spatial maxima, resulting from the three waves coupling, are representative of the localization of Raman activity. The propagation of those maxima is represented for different values of $\eta$ and for the PIC simulation. We

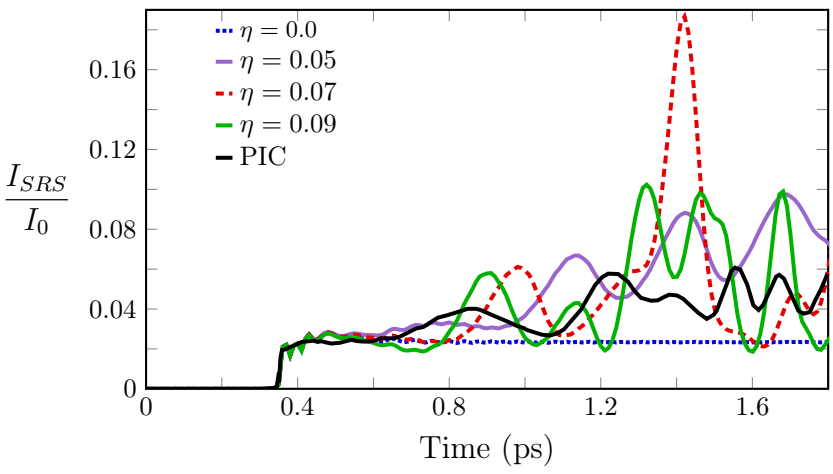

Figure 9. Comparison of the PIC reflectivity (black curve) and reflectivity given by fluid-like model for different $\eta$ values: 0 (blue dotted), $5 \%$ (purple), $7 \%$ (red dash) and 9\% (green).

see that the best match is for $\eta=0.07$, that is in agreement with the estimation (28) from the PIC distribution function. The case $\eta=0$ corresponds to a case without non-linear effects. The resonant point is set by the initial conditions and geometry (here at $x_{\|}=5 \mu \mathrm{m}$ ) and does not evolve through time. In this case, the EPW amplitude is driven by the Rosenbluth gain inside the turning points and stays very small. The detection of the first maximum algorithm was not designed for this case and catches here modulations due to the numerical scheme which explains why it evolves a little bit before the stationary case is reached. Though, we chose not to modify the algorithm in order to use the exact same post-processing management.

In these simulations, 2D effects such as EPW selffocusing, EPW filamentation or the curvature of the phase front are expected to occur. In the PIC simulations we can identify the phenomenon of wave front bowing ${ }^{15}$. In the simulations with the wave-coupling code SIERA, curvature in the phase front is also observable, while not resolving the EPW wavelength. However, the curvature of the EPW front is very similar to the one observed in the PIC simulation once post processed with the Hilbert transform to extract the wave envelope. In Fig. 11 and 12, we present at two different times the EPW amplitudes obtained with the PIC code (top), its envelope extraction using the previously presented method (middle) and the envelope calculated with the paraxial model (bottom), for $\eta=0.07$. The comparison shows that the fluid code catches the most important specifications: activity area, spatial extension, global shape, filamentation, islets in the wave head, self-focusing and the spatial mean intensity. This translates directly the efficiency of the wave coupling (growth, enhancement regarding the Rosenbluth case and saturation) and so the intensity of the Raman wave. Note that we use a paraxial solver in the fluid code SIERA, this could explain some remaining differences. The comparison of the different structures around the resonant point $(z=0)$ is very well described for $\eta=0.07$. 


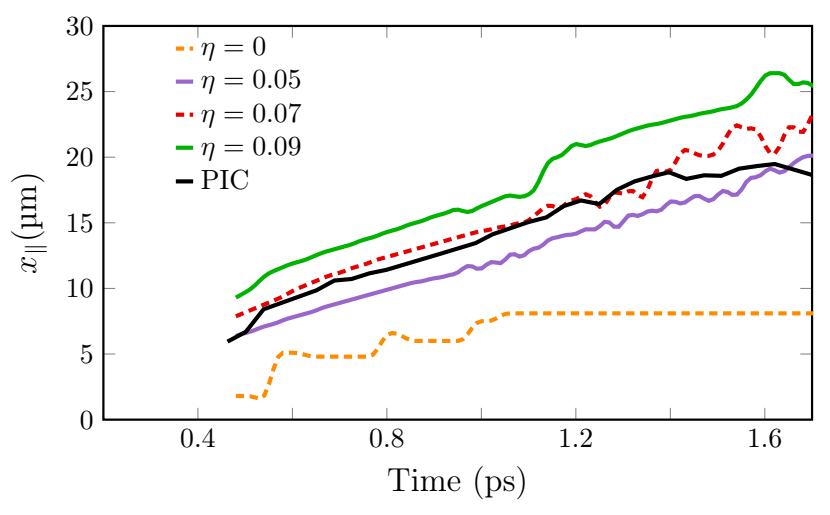

Figure 10. Comparison of the position of first maximum of EPW given by PIC (black) code and the same given by fluid-like model for different $\eta$ values: $0 \%$ (orange dash), $5 \%$ (purple), $7 \%$ (red dash) and $9 \%$ (green).

\section{DISCUSSION AND CONCLUSION}

The effective cost of each 2D PIC simulation is roughly 170 hours times 2,048 cpus, whereas the simulation duration of the fluid code is one hour on a desktop computer. So, given this economy of time and means, and the quite good comparisons obtained, this approach is really encouraging particularly when considering the relative simplicity (theoretical and numerical) of the model. Of course there is still the free parameter $\eta$ to be adjusted. But, as it can be seen on the different figures presented above, the system is not so sensitive. Few more configurations ${ }^{46}$ were successfully tested regarding the intensity of the pump wave, the geometry, and the plasma density slab $L_{\nabla}$.

The aim of such a model is to obtain quickly a good order of magnitude of the Raman scattered wave for a given set of plasma parameters in the domain of applicability of the model. So a strategy could be to make several runs with different but close values for $\eta$ in order to determine in which interval relies the response of the system. This kind of model is also relevant for dealing with large (millimeter size) system over a long time scale (hundreds of picoseconds) which is not the case of PIC codes. Furthermore, as the model is based on a parametrization of the electron function distribution (7), it is possible to build back a distribution function with the calculated parameter $\delta$ and to compare it with the distribution given by the PIC code. In Fig. 13 we present such a calculated electron distribution function that compares quite well to the PIC distribution function presented in Fig. 8.

As mentioned above, side effects could probably play an important role for SRS. This is discussed by ${ }^{21,22}$ and that is why we use a wide gaussian pump $\left(f_{\#}=16\right)$. Good agreements were also obtained with a wider gaussian beam $\left(f_{\#}=30\right)$. In these configurations, $2 \mathrm{D}$ effects are not expected to be too strong. For studying more realistic cases (for example the interaction between speckles in the case of a smoothed beam), the modelling
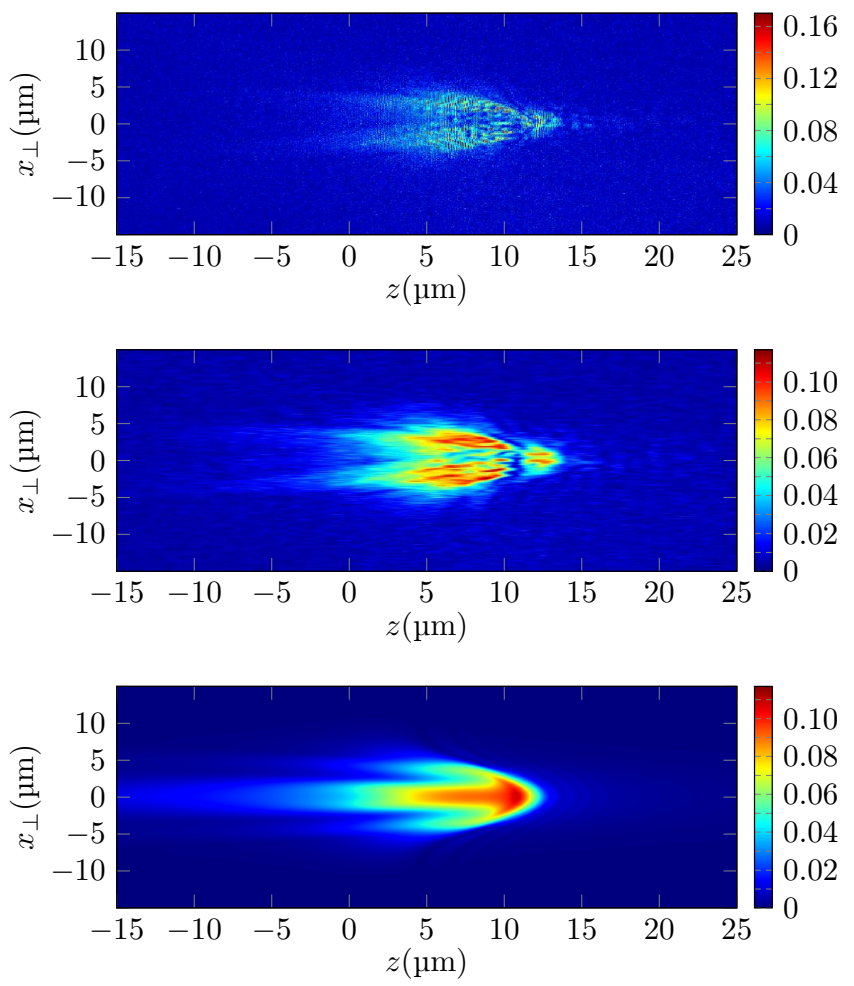

Figure 11. (Inhomogeneous plasma case) EPW field in $\left|\frac{\delta n_{e}}{N_{0}}\right|$ at $4,800 \omega_{0} t=0.8$ ps. Top: data given by the PIC code EMI2D. Middle: Envelope of the above, corresponding to the EPW field from the PIC simulation, around the linear Raman mode, extracted with the Hilbert transform ${ }^{13}$. Bottom: Envelope of the EPW calculated with the fluid-like model for $\eta=0.07$.

of transverse effects will be needed. Other non linear mechanisms such as electron detrapping should also be considered in a future work.

In conclusion, we developed a reduced model in the paraxial approximation for SRS in a regime where kinetic effects due to electron trapping are expected to be important. The kinetic effects are calculated from a dynamical model for the electron distribution function which is parametrized with the width of the plateau associated to the flattening around the phase velocity. This model was compared with PIC simulations in configurations that allowed us to perfectly control the linear response of the system.

The results compare very well to PIC code simulations. However, the model is not fully predictive in the sense that a free parameter $\eta$ has to be adjusted to best reproduce PIC results. But the system is not that sensitive to this parameter, so different runs with different values for $\eta$ would give tendencies of the system response. Contrary to the model by Morales and O'Neil, which suppose that the deformation of the distribution function is instantly established to its final state, this model 

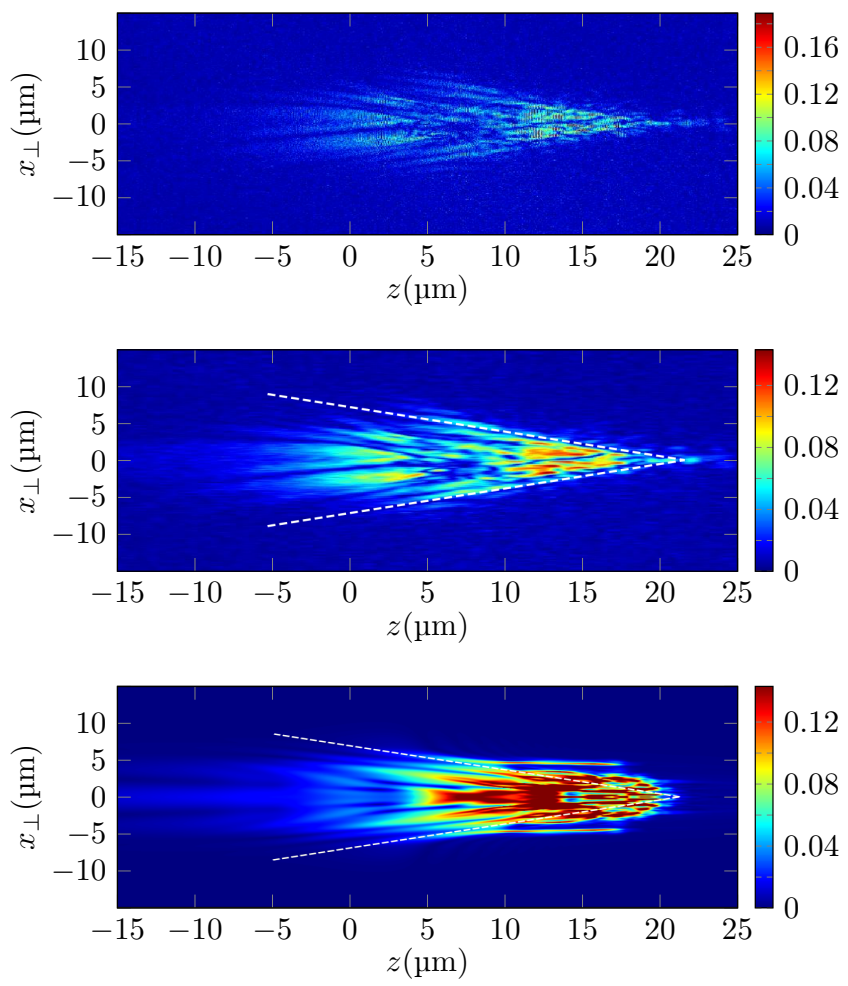

Figure 12. (Inhomogeneous plasma case) EPW field in $\left|\frac{\delta n_{e}}{N_{0}}\right|$ at $8,600 \omega_{0} t=1.60$ ps. Top: Data given by the PIC code EMI2D. Middle: Envelope of the above signal, around the linear Raman mode, extracted with the Hilbert transform ${ }^{13}$. Bottom: Envelope of the EPW calculated with the fluid-like model for $\eta=0.07$. The spatial extension is quite well described, particularly the angle of the filaments (around $14^{\circ}$, which is still compatible with the paraxial approximation).

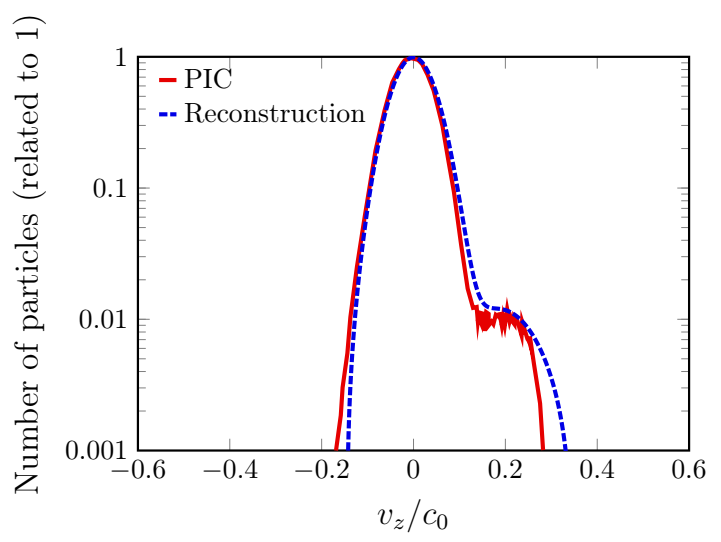

Figure 13. Reconstruction of the distribution function (green) compared to the PIC distribution function (red) at 1.5 ps with $\eta=0.08$.

gives a frequency shift for a distribution function during its deformation. This point is essential as the original "static" model couldn't reproduce PIC simulations with enough precision. Due to its relative simplicity, such modelling may be implemented in massively parallel, 3D paraxial interaction codes, such as HERA ${ }^{40}$. Using this model would allow performing simulations including more physics, e.g. hydrodynamics, Brillouin scattering and possibly the competition with SRS, using optically smoothed beams. In addition, such modeling may prove to be useful in wider applications such as shock ignition studies where non linear kinetic effects are wanted, but also in Raman amplification schemes where pump-probe laser systems are used with high-intensity lasers. Nevertheless, this model could still be enhanced by adding a description of transverse effects and other mechanisms such as detrapping.

\section{ACKNOWLEDGMENTS}

This work has been partially supported by the Agence Nationale de la Recherche, project title "Ilphygerie" no. ANR-12-BS04-0006. This work was granted access to the HPC ressources of IDRIS under the allocation 2014/2016-0500573 made by GENCI. The authors also thank Denis Pesme, Michel Casanova, Stephan Brunner, Patrick Mora, Alain Ghizzo for helpful discussions.

\section{DATA AVAILABILITY}

The data that support the findings of this study are available from the corresponding author upon reasonable request.

\section{REFERENCES}

${ }^{1}$ S. H. Glenzer, B. K. Spears, M. J. Edwards, E. T. Alger, R. L. Berger, D. L. Bleuel, D. K. Bradley, J. A. Caggiano, D. A. Callahan, and C. C. et. al., Plasma Phys. Control. Fusion 54, 045013 (2012).

${ }^{2}$ C. Rousseaux, G. Huser, P. Loiseau, M. Casanova, E. Alozy, B. Villette, R. Wrobel, O. Henry, and D. Raffestin, Phys. Plasmas 22, 022706 (2015).

${ }^{3}$ J. F. Drake, P. K. Kaw, Y. C. Lee, G. Schmidt, C. S. Liu, and M. N. Rosenbluth, Phys. Fluids 17, 778 (1974).

${ }^{4}$ H. X. Vu, D. F. Dubois, and B. Bezzerides, Phys. Plasmas 9, $1745(2002)$.

${ }^{5}$ J. L. Kline, D. S. Montgomery, L. Yin, D. F. DuBois, B. J. Albright, B. Bezzerides, J. A. Cobble, E. S. Dodd, D. F. DuBois, J. C. Fernàndez, R. P. Johnson, J. M. Kindel, and H. A. Rose, Phys. Plasmas 13, 055906 (2006).

${ }^{6}$ T. Kolber, W. Rozmus, and V. T. Tikhonchuk, Phys. Fluids B 5, 138 (1993).

${ }^{7}$ T. Kolber, W. Rozmus, and V. T. Tikhonchuk, Phys. Rev. Lett. 70, 1810 (1993).

${ }^{8}$ W. L.Kruer, The physics of laser plasma interactions AddisonWesley (1988).

${ }^{9}$ B. L. Fontaine, D. M. Villeneuve, H. A. Baldis, R. P. Drake, and K. Estabrook, Phys. Rev. Lett. 68, 484 (1992).

${ }^{10}$ R. L. Dewar, Phys. Fluids 15, 712 (1972).

${ }^{11}$ R. L. Dewar and J. Lindl, Phys. Fluids 15, 820 (1972).

${ }^{12}$ G. J. Morales and T. M. O'Neil, Phys. Rev. Lett. 28, 417 (1972).

${ }^{13}$ R. L. Berger, S. Brunner, T. Chapman, L. Divol, C. H. Still, and E. J. Valeo, Phys. Plasmas 20, 032107 (2013). 
${ }^{14}$ L. Yin, W. Daughton, B. J. Albright, K. J. Bowers, D. S. Montgomery, J. L. Kline, J. C. Fernàndez, and Q. Roperd, Phys. Plasmas 13, 072701 (2006).

${ }^{15}$ L. Yin, B. J. Albright, K. J. Bowers, W. Daughton, and H. A. Rose, Phys. Plasmas 15, 013109 (2008).

${ }^{16}$ L. Yin, B. J. Albright, H. A. Rose, K. J. Bowers, B. Bergen, D. S. Montgomery, J. L. Kline, and J. C. Fernàndez, Phys. Plasmas 16, 113101 (2009).

${ }^{17}$ L. Yin, B. J. Albright, H. A. Rose, K. J. Bowers, B. Bergen, R. K. Kirkwood, D. E. Hinkel, A. B. Langdon, P. Michel, D. S. Montgomery, and J. L. Kline, Phys. Plasmas 19, 056304 (2012).

${ }^{18}$ L. Yin, B. J. Albright, H. A. Rose, D. S. Montgomery, J. L. Kline, R. K. Kirkwood, P. Michel, K. J. Bowers, and B. Bergen, Phys. Plasmas 20, 012702 (2013).

${ }^{19}$ L. Yin, B. J. Albright, H. A. Rose, D. S. Montgomery, J. L. Kline, R. K. Kirkwood, J. Milovich, S. M. Finnegan, B. Bergen, and K. J. Bowers, Phys. Plasmas 21, 092707 (2014).

${ }^{20}$ P.-E. Masson-Laborde, W. Rozmus, Z. Peng, D. Pesme, S. Hüller, M. Casanova, V. Y. Bychenkov, T. Chapman, and P. Loiseau, Phys. Plasmas 17, 092704 (2010).

${ }^{21}$ S. Brunner, R. L. Berger, B. I. Cohen, L. Hausammann, and E. J. Valeo, Phys. Plasmas 21, 102104 (2014).

${ }^{22}$ R. L. Berger, S. Brunner, J. W. Banks, B. I. Cohen, and B. J. Winjum, Phys. Plasmas 22, 055703 (2015).

${ }^{23}$ Y. X. Wang, Q. S. Feng, H. C. Zhang, Q. Wang, C. Y. Zheng, Z. J. Liu, and X. T. He, Phys. Plasmas 24, 103122 (2017).

${ }^{24}$ Y. X. Wang, Q. Wang, C. Y. Zheng, Z. J. Liu, Q. S. Feng, C. S. Liu, and X. T. He, Plasma Phys. Control. Fusion 62, 075009 (2020).

${ }^{25}$ H. X. Vu, D. F. Dubois, and B. Bezzerides, Phys. Plasmas 14, 012702 (2007).

${ }^{26}$ S. M. Finnegan, L. Yin, J. L. Kline, B. J. Albright, and K. J. Bowers, Phys. Plasmas 18, 032707 (2011).

${ }^{27}$ A. Friou, D. Bénisti, L. Gremillet, E. Lefebvre, O. Morice, E. Siminos, and D. J. Strozzi, Phys. Plasmas 20, 103103 (2013).

${ }^{28}$ R. R. Lindberg, A. E. Charman, and J. S. Wurtele, Phys. Plasmas 15, 055911 (2008).

${ }^{29}$ N. A. Yampolsky and N. J. Fisch, Phys. Plasmas 16, 072104 (2009).

${ }^{30}$ N. A. Yampolsky and N. J. Fisch, Phys. Plasmas 16, 072105 (2009).

${ }^{31}$ T. Chapman, S. Hüller, P. E. Masson-Laborde, W. Rozmus, and D. Pesme, Phys. Plasmas 17, 122317 (2010).

${ }^{32}$ T. Chapman, S. Hüller, P. E. Masson-Laborde, A. Héron, D. Pesme, and W. Rozmus, Phys. Rev. Lett. 108, 145003 (2012).

${ }^{33}$ T. Chapman, S. Hüller, P. Masson-Laborde, A. Heron, W. Rozmus, and D. Pesme, EPJ Web of Conferences 59, 05012 (2013).
${ }^{34}$ T. P. Coffey, Phys. Fluids 14, 1402 (1971).

${ }^{35}$ B. J. Winjum, J. Fahlen, and W. B. Mori, Phys. Plasmas 14, 102104 (2007).

${ }^{36}$ O. Yaakobi, L. Friedland, R. R. Lindberg, A. Charman, G. Penn, and J. S. Wurtele, Phys. Plasmas 15, 032105 (2008).

${ }^{37}$ H. A. Rose and L. Yin, Phys. Plasmas 15, 042311 (2008).

${ }^{38}$ R. L. Berger, C. H. Still, E. A. Williams, and A. B. Langdon, Phys. Plasmas 5, 4337 (1998).

${ }^{39}$ S. Hüller, P. E. Masson-Laborde, D. Pesme, M. Casanova, F. Detering, and A. Maximov, Phys. Plasmas 13, 022703 (2006).

${ }^{40}$ P. Loiseau, O. Morice, D. Teychenné, M. Casanova, S. Hüller, and D. Pesme, Phys. Rev. Lett. 97, 205001 (2006).

${ }^{41}$ P. Ballereau, M. Casanova, F. Duboc, D. Dureau, H. Jourdren, P. Loiseau, J. Metral, O. Morice, and R. Sentis, J. Scient. Comput. 33, 1 (2007).

${ }^{42}$ P. Loiseau, P.-E. Masson-Laborde, D. Teychenné, M.-C. Monteil, M. Casanova, D. Marion, G. Tran, G. Huser, C. Rousseaux, S. Hüller, A. Héron, and D. Pesme, J. Phys.: Conf. Ser. 688, 012059 (2016).

${ }^{43}$ D. Bénisti, O. Morice, L. Gremillet, A. Friou, and E. Lefebvre, Phys. Plasmas 19, 056301 (2012).

${ }^{44}$ D. Bénisti, Plasma Phys. Control. Fusion 60, 014040 (2018).

${ }^{45}$ D. J. Strozzi, E. A. Williams, H. A. Rose, D. E. Hinkel, A. B. Langdon, and J. W. Banks, Phys. Plasmas 19, 112306 (2012).

${ }^{46}$ G. Tran, PhD Thesis in french (2015), Étude et modélisation de la diffusion Raman stimulée en configuration multidimensionnelle pour la FCI. https://pastel.archivesouvertes.fr/tel-01264367.

${ }^{47}$ J. C. Adam, A. G. Servenière, , and A. B. Langdon, J. Comput. Phys. 47, 229 (1989).

48 A. Héron and J.-C. Adam, Phys. Plasmas 22, 072306 (2015).

${ }^{49}$ M. N. Rosenbluth, Phys. Rev. Lett. 29, 565 (1972).

${ }^{50}$ L. Divol, E. A. Williams, B. I. Cohen, A. B. Langdon, and B. F. Lasinski, Proc. IFSA 2003, 233 (2003).

${ }^{51}$ E. A. Williams, B. I. Cohen, L. Divol, M. R. Dorr, J. A. Hittinger, D. E. Hinkel, A. B. Langdon, R. K. Kirkwood, D. H. Froula, and S. H. Glenzer, Phys. Plasmas 11, 231 (2004).

${ }^{52} \mathrm{P}$. communication, .

${ }^{53}$ D. Bénisti, N. A. Yampolsky, and N. J. Fisch, Phys. Plasmas 19, 013110 (2012).

${ }^{54}$ P. Mounaix, D. Pesme, and M. Casanova, Phys. Rev. E 55, 4653 (1997).

55 Note that the Rosenbuth gain is defined in 1D geometry. It can be generalized to higher geometrical dimensions by a transverse integration. 